\title{
The antibacterial efficacy of a foam mouthwash and its ability to remove biofilms
}

Siân B. Jones ${ }^{1}$, Nicola X. West ${ }^{1}$, Pavel P. Nesmiyanov ${ }^{2,3}$, Sergey E. Krylov ${ }^{4,5}$, Vera V. Klechkovskaya ${ }^{5}$, Natalya A. Arkharova ${ }^{5}$ and Svetlana A. Zakirova ${ }^{6}$

OBJECTIVES/AIMS: To evaluate the antimicrobial activity of a newly developed foam mouthwash containing a modified lactoperoxidase system in vitro.

MATERIALS AND METHODS: Biofilms of five bacterial species were developed on hydrophobic and hydrophilic surfaces whilst salivary-based biofilm was grown on tooth enamel. Each surface was exposed to the foam mouthwash or saline in vitro. Optical density and scanning electron microscopy (SEM) was used to determine retention of the biofilm following 5 or $30 \mathrm{~s}$ exposure time. RESULTS: The foam mouthwash was active against biofilms formed by S. aureus, K. rhizophila, M. thailandicus, E. coli, and C. violaceum and eliminated significant amount of biofilm from each surface; immature $4 \mathrm{~h}$ biofilm was less resistant than $24 \mathrm{~h}$ biofilm. A $30 \mathrm{~s}$ rinse showed best performance, with removal of up to $66 \%$ of biofilm from the hydrophilic surface. SEM imaging confirmed oral biofilm removal from the enamel surface after a $5 \mathrm{~s}$ rinse with the foam mouthwash.

DISCUSSION: Foam mouthwash demonstrated a significant impact on growing biofilm when compared against saline solution. Growing biofilms were more susceptible to the action of the foam mouthwash, which justifies after-meal use of the mouthwash when traditional dentifrices may not be accessible.

CONCLUSIONS: Foam mouthwash can be a convenient on-the-go format of oral care products that can be used after meals or when needed to reduce the risk of biofilm-associated oral conditions.

BDJ Open (2018)4:17038; https://doi.org/10.1038/s41405-018-0005-5

\section{INTRODUCTION}

Biofilms are complex microbial communities characterized by cells attached to the substrate surface, to interfaces or to each other and are embedded in an extracellular polymeric matrix which they have produced. ${ }^{1-3}$ Mixed culture microbial biofilms comprise dental plaque that can be beneficial to the host, but biofilms are often associated with diseases. ${ }^{4}$ Tightly adherent mature biofilms are a primary cause of caries and their formation needs to be prevented. ${ }^{5}$ Clinically biofilms form on native tissues such as oral mucosa and teeth and often cause chronic infection of dental implants. ${ }^{6-8}$ The behavior of microbes in a biofilm can differ significantly from the behavior of the same organism studied in planktonic conditions. ${ }^{3}$ Most studies on dentifrices focus on bacteria commonly isolated from dental plaque, however these studies do not take into consideration the different adhesion mechanisms of some bacterial species and the beneficial role of some microorganisms implicated in biofilm formation. ${ }^{9,10}$

Latest data on human microbiome suggest that best oral care practices should not seek complete elimination of biofilms but to control their formation without damaging the protective pellicle. $^{11}$ One of the possibilities to beneficially alter the ecology of dental biofilm is the use of lactoperoxidase system. ${ }^{12}$ Lactoperoxidase systems have been incorporated into dental healthcare products such as dentifrice and mouthwashes since the 1980s. ${ }^{13}$ The byproduct of lactoperoxidase is hypothiocyanate which acts as a natural antibacterial agent. ${ }^{12}$ The management of biofilm related conditions can be problematic as the structure and composition of the biofilm itself offers protection against antimicrobial agents and frequently conjunctive mechanical biofilm disruption is required to enable surface disinfection. $^{14}$

The effectiveness of over-the-counter toothpastes is without question but toothpastes have limited portability and are not well suited for daytime use. At the same time, maturing biofilms are most susceptible to external impacts, which justifies the use of oral care products during the day, especially after meals. Considerable research effort is currently being spent developing portable products for on-the-go use. Conventional mouthwashes cannot be attributed to easy-to-use formats because of relatively large package volumes and high consumption volume (about 20 $\mathrm{mL}$ ). A novel format of mouthwashes has recently been developed in the form of a foam mouthwash, which is portable and convenient to use. The aim of the present in vitro study was to investigate the antibacterial effect of foam mouthwash against biofilms formed on the surfaces mimicking real-life conditions. The null hypothesis being that a foam mouthwash has no greater effect than saline at removing biofilm.

\footnotetext{
${ }^{1}$ Clinical Trials Unit, Bristol Dental School, Bristol, UK; ${ }^{2}$ Fundamental Medicine and Biology Department, Volgograd State Medical University, Volgograd, Russian Federation; ${ }^{3}$ Department of Immunology, Biological Faculty, Lomonosov Moscow State University, Moscow, Russian Federation; ${ }^{4}$ LLC BITECA, Odintsovo, Moscow region, Moscow, Russian

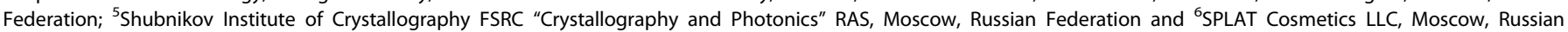
Federation

Correspondence: Pavel P. Nesmiyanov (pecatum@yandex.ru)
} 
Table 1 Growing $(4 \mathrm{~h})$ and mature $(24 \mathrm{~h})$ biofilm retention (O.D. 590, $5 \mathrm{~s} / 30 \mathrm{~s}$, data presented as median, $\%$ to control $($ Control $=100 \%))$

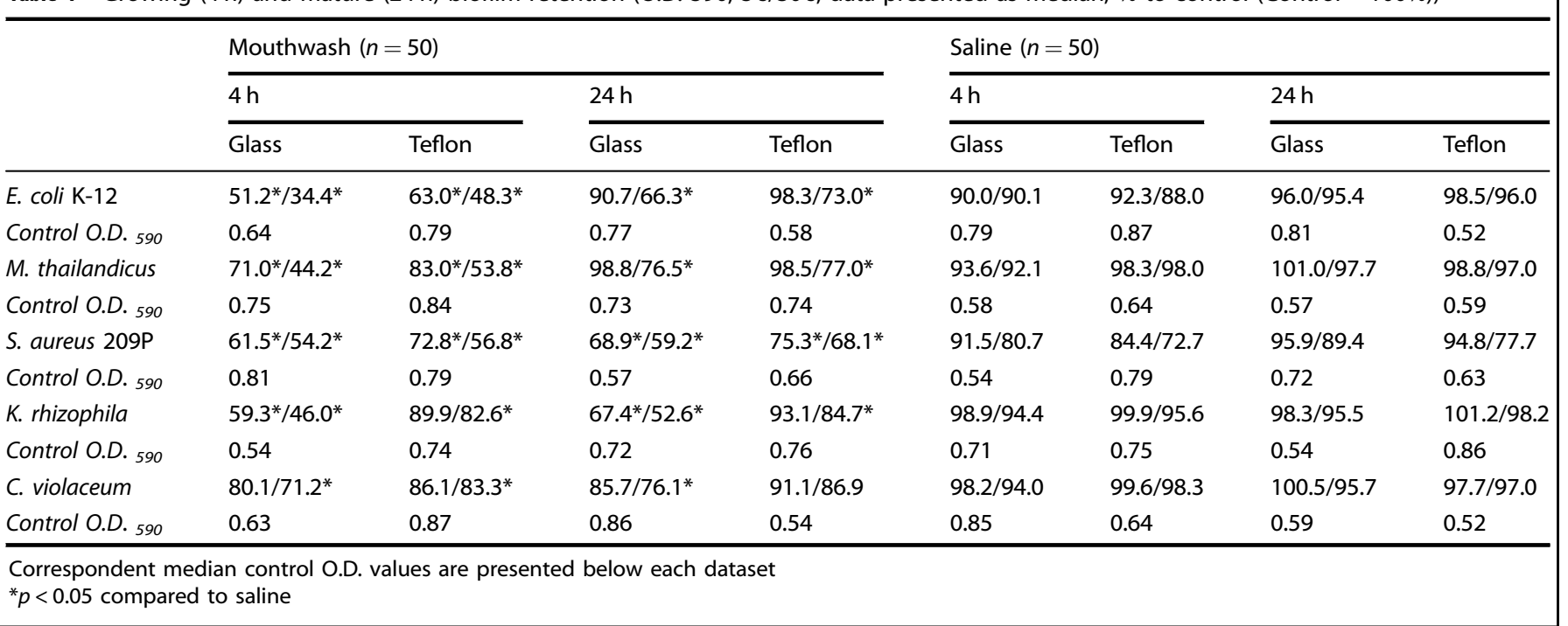

\section{MATERIALS AND METHODS}

This study was conducted in two parts. One in which artificial surfaces were used for biofilm quantification and another where extracted teeth were used for visualization by scanning electron microscopy (SEM).

\section{Solutions and products used}

"Splat Oral Care Foam Raspberry 2 in $1^{\mathrm{Tm} \text { " }}$ (SPLAT Cosmetics, Russia) mouthwash was used. This product contains modified lactoperoxidase system [potassium thiocyanate, lactoferrin, lactoperoxidase, glucose oxidase, glucose pentaacetate, Glycirrhizia Glabra root extract] named as the registered trademark Luctatol ${ }^{\mathrm{TM}}$ and detergents, designed to provide cleaning and antibacterial action. Sterile saline was used as control.

Biofilm retention study

Several strains of bacteria to model biofilm formation on the surfaces were used. Gram-positive Staphylococcus aureus 209P, Kocuria rhizophila 4A-2ZH (also known as Micrococcus luteus), Micrococcus thailandicus HB, Gram-negative Escherichia coli K-12 and Chromobacterium violaceum WT were obtained from the strain collections of the research center of biotechnology RAS (Moscow, Russia). These bacteria were selected on the basis of their different adhesion mechanisms and different extracellular matrix structure. Bacteria were seeded onto solid LB medium (Carl Roth $\mathrm{GmbH}$, Karlsruhe, Germany) with consequent transfer of single colonies into liquid LB medium and incubated for $24 \mathrm{~h}$ at $30^{\circ} \mathrm{C}$. Teflon cubes ( $n=10$ for each condition) were used for biofilm formation, as described in Plakunov et al. ${ }^{15}$ Cubes were immersed in LB medium containing $50 \mu \mathrm{L}$ of $24 \mathrm{~h}$ culture diluted to $2 \times 10^{9} \mathrm{CFU} / \mathrm{mL}$ and incubated for 4 and $24 \mathrm{~h}$ at $30^{\circ} \mathrm{C}$ on a MaxQ 4000 rocker platform (150 rpm) (Thermo Scientific, Waltham, MA, USA) allowing for biofilms to be deposited both on the cubes and vial glass internal surface (to mimic tooth enamel hydrophobic and hydrophilic properties). LB without bacteria was used as a blank. After incubation, cubes and vials were washed with sterile saline and washed again with $3 \mathrm{~mL}$ mouthwash or saline for 5 or 30 seconds using a vortex (Micro-Spin FV-2400, BioSan, Lithuania) set at $3000 \mathrm{rpm}$. Unwashed samples were used as a control and the amount of biofilm retention on the treated surfaces was quantified as a $\%$ difference from this value. Biofilms were then stained with Crystal Violet (Sigma, St Louis, MO, USA) for $30 \mathrm{~min}$. After washing with distilled water the biofilms were extracted from cubes and vials surfaces with $96 \% \mathrm{EtOH}$ for $1 \mathrm{~h}$.
Optical density of the resulting solution was measured at $590 \mathrm{~nm}$ using a Statfax 2100 optical density reader (Awareness Technology, Palm City, FL, USA).

Biofilm retention and SEM imaging study

The ethics committee of Shubnikov Institute of Crystallography FSRC "Crystallography and Photonics" RAS approved the experimental protocol for the use of human teeth and saliva (Protocol \#7). Extracted mandibular central incisors $(n=10)$ were cleaned with a hard toothbrush and high-abrasion silica (Sorbosil ac33 ${ }^{\circledR}$, Surfachem, Leeds, UK) before being ultrasonically cleaned in distilled water for $4 \mathrm{~h}$ at $35 \mathrm{kHz}$ (Sonoswiss SW3H, Sonoswiss AG, Ramsen, Switzerland). Teeth were air-dried at room temperature. After this cleaning method the teeth showed no signs of bacterial contamination under SEM.

After primary SEM imaging the teeth were transferred into $20 \mathrm{~mL}$ A. C. Broth (Sigma, St Louis, MO, USA) containing pooled human saliva and incubated for $48 \mathrm{~h}$ at $37^{\circ} \mathrm{C}$ on a MaxQ 4000 rocker platform. After $24 \mathrm{~h}$, a change in $\mathrm{pH}$ from 6.8 to 3.5 was noted and the medium was replaced with fresh medium and incubated for another $24 \mathrm{~h}$, as described by Zhou et al. ${ }^{16}$

Saliva was pooled for wild-type organisms from five subjects who refrained from all oral hygiene procedures overnight. Pooled tongue scrapings from five different individuals were also obtained using tongue cleaners which were rinsed with $0.01 \%$ peptone. The samples were then centrifuged for $10 \mathrm{~min}$ at $10,000 \times g$ (MicroCL 21R, Thermo Scientific, Waltham, MA, USA), following which the pellets were resuspended in $A$. C. Broth to a final volume of $20 \mathrm{~mL}$. Pre-study criteria for saliva pooling was that donors did not consume any antibiotic products in the month previous to the sampling day, refrained from using an antiseptic mouthwash in the week before the study, avoided any oral hygiene measures in the morning when the sample was taken and abstained from food or drink intake for at least $2 \mathrm{~h}$ prior to donating saliva. Second SEM analysis was made after the incubation to detect biofilm formation.

Five incisors were agitated using a vortex (Micro-Spin FV-2400, BioSan, Lithuania) set at $3000 \mathrm{rpm}$ in $5 \mathrm{~mL}$ foam mouthwash for $5 \mathrm{~s}$ and 5 incisors were incubated in sterile saline for $5 \mathrm{~s}$. After incubation the teeth were rinsed with distilled water and air-dried at room temperature before third SEM analysis.

SEM was carried out on the surfaces of uncoated samples attached to carbon tabs on a Scios field emission scanning electron microscope (FEl, Eindhoven, The Netherlands), operated 



Fig. 1 Representative SEM images of enamel surfaces. a-c After biofilm formation, before foam or saline rinsing; a1, b1—after foam rinsing, c1 -after saline rinsing. Black arrows indicate biofilm location site. White arrows indicate erosive pits

at an accelerating voltage of $1.0 \mathrm{kV}$. Samples were placed into the SEM sample holder at the same orientation each time so that the same area could be identified and standardized. Imaging was performed at a working distance of $5-7 \mathrm{~mm}$. Images taken at $\times 8000$ and $\times 30,000$ magnification was used for analysis.

Statistical analysis

The statistical analysis was performed using IBM SPSS 22 for Windows. To detect statistically significant inhibition in an unbiased approach, we performed the non-parametric sign-test. The level of significance was set at 0.05 .

\section{RESULTS}

Biofilm removal

Results on the $E$. coli and M. thailandicus biofilm removal are presented in Table 1. Our data suggest that foam mouthwash was superior to saline in the removal of both growing and mature 
biofilms. However, in most cases 5 -s rinse was not enough to remove mature biofilm. Similar results were obtained with $S$. aureus and $K$. rhizophila biofilms. However, in all cases foam mouthwash reduced growing biofilm significantly after a 30-s rinsing procedure, being most effective on glass surface. $C$. violaceum biofilms was most resistant to rinsing while $E$. coli was most susceptible with up to $2 / 3$ growing biofilms removed (34.4\% retention) after a 30 -s rinse.

\section{Scanning electron microscopy}

SEM images of the enamel surface captured prior to biofilm formation (not shown) appeared smooth and clean. There were no bacteria, pellicle, particles, or deposits. Surfaces without carious lesions or demineralization were used.

After incubation with pooled saliva, biofilms were formed at the enamel surfaces and enamel erosions were present. As shown in Fig. 1, a 5-s rinse with foam mouthwash led to significant removal of biofilms compared to saline control.

\section{DISCUSSION}

The present study investigated the effectiveness of foam mouthwash rinsing on biofilm retention on different surfaces. The study design was developed to explore the capability of foam mouthwash to remove biofilms of bacteria with different adhesion properties from diverse surfaces-hydrophilic (glass), hydrophobic (Teflon cubes), and tooth enamel. Foam mouthwashes are a relatively new type of oral care product on the market and there are no previously published studies on the efficacy of such products. Fluoridated foams used for caries control have previously been shown to reduce the case of caries development when compared to the more commonly used fluoride gels. ${ }^{17}$ Foams are deemed advantageous because of the reduced amount of volume that is required to achieve the same outcome as when fluoridated gels are used. ${ }^{18}$ Most mouthwashes claim the ability to clean teeth after 5-30 s swishing. This ability is mostly mediated by surfactants that change the permeability of bacterial cell membrane with subsequent cell lysis. ${ }^{19,20}$ The presence of the foam pump enables output of the mouthwash liquid in the form of a foam with bubble size ranging from 0.05 to $0.5 \mathrm{~mm}$. This allows a reduced mouthwash volume to be used whilst more importantly, employs the physical phenomenon of bacterial cell damage by the bubble rupture at the gas-liquid interface. ${ }^{21,22}$

In both parts of this study the foam mouthwash demonstrated a significant impact on growing biofilm when compared against saline solution. The first part of the study showed that $E$. coli was most susceptible and $C$. violaceum was most resistant to the action of mouthwash. As expected, growing, or immature $4 \mathrm{~h}$ biofilms were more susceptible to the action of the foam mouthwash, which justifies after-meal use of the mouthwash when traditional dentifrices may not be accessible. In addition, since recommended use of mouthwash does not imply water rinsing, it is expected that the lactoperoxidase system within the foam mouthwash will continue to work for a certain period of time, thus preventing colonization and cariogenic action of acid-producing bacteria. ${ }^{12,23}$

The results obtained from the second part of the study where enamel was used as substrate again showed that the foam mouthwash performed better than saline at removing biofilm. The biofilm formed on the enamel surface was a mature biofilm as the method of growing the biofilm meant that a $48 \mathrm{~h}$ biofilm was developed. During the development of the biofilm, erosive pits also formed on the surface. The erosive pits were not altered by the exposure to the foam mouthwash. The $\mathrm{pH}$ of the foam mouthwash was 7.4 which is similar to Bioténe Oral Balance which also contains lactoperoxidase and has previously been shown to not cause enamel erosion. ${ }^{24}$ The SEM images show that the biofilm was not uniformly developed over the surface but that the amount removed following a $5 \mathrm{~s}$ rinse with the foam mouthwash was greater than with saline. Increasing the exposure time to $30 \mathrm{~s}$ would most likely remove more of the formed biofilm as evidenced in the first part of the study. Interestingly, the amount of mature $(24 \mathrm{~h})$ biofilm removed from the glass and Teflon substrates after a $5 \mathrm{~s}$ treatment with the foam mouthwash was not great. Only the S. aureus $209 \mathrm{P}$ and the K. rhizophila showed significant removal compared to saline. This highlights the importance of recognizing that differently formed biofilms can provide varying results and that the type of substrate used can also determine the amount of biofilm retention.

\section{CONCLUSIONS}

In conclusion, the results from this study can reject the null hypothesis as the foam mouthwash was significantly more effective than saline at removing biofilm from each of the surfaces investigated. The foam mouthwash can be recommended as a convenient, additional oral hygiene device for daily after meal use.

\section{ACKNOWLEDGEMENTS}

N.X.W., P.P.N., S.A.Z. conceived and designed the experiments. S.E.K., N.A.A., V.V.K. performed the SEM imaging. S.B.J., N.X.W., P.P.N. wrote the paper. This work was supported by the Federal Agency for Scientific Organizations (Agreement No 007-ГЗ/ 43363/26) and performed using the equipment of the Shared Research Center FSRC "Crystallography and photonics" RAS and was supported by the Russian Ministry of Education and Science.

\section{ADDITIONAL INFORMATION}

Competing interests: P.P.N. and S.A.Z. are employed by SPLAT-Cosmetics LLC. The remaining authors declare no competing interests.

Publisher's note: Springer Nature remains neutral with regard to jurisdictional claims in published maps and institutional affiliations.

\section{REFERENCES}

1. Costerton, J. W., Geesey, G. G. \& Cheng, K. J. How bacteria stick. Sci. Am. 238, 86-95 (1978).

2. Costerton, J. W., Stewart, P. S. \& Greenberg, E. P. Bacterial biofilms: a common cause of persistent infections. Science 284, 1318-1322 (1999).

3. Donlan, R. M. \& Costerton, J. W. Biofilms: survival mechanisms of clinically relevant microorganisms. Clin. Microbiol. Rev. 15, 167-193 (2002).

4. Marsh, P. O. \& Bradshaw, O. J. Microbiological effects of new agents in dentifrices for plaque control. Int. Dent. J. 43, 399-406 (1993).

5. Jeon, J. G., Rosalen, P. L., Falsetta, M. L. \& Koo, H. Natural products in caries research: current (limited) knowledge, challenges and future perspective. Caries Res. 45, 243-263 (2011).

6. Bjarnsholt, T. The role of bacterial biofilms in chronic infections. APMIS 121, 1-58 (2013).

7. Clegg, M. S., Vertucci, F. J., Walker, C., Belanger, M. \& Britto, L. R. The effect of exposure to irrigant solutions on apical dentin biofilms in vitro. J. Endod. 32, 434-437 (2006)

8. Schwarz, F. et al. Influence of plaque biofilm removal on reestablishment of the biocompatibility of contaminated titanium surfaces. J. Biomed. Mater. Res. A 77, 437-444 (2006).

9. Finney, M., Walker, J. T., Marsh, P. D. \& Brading, M. G. Antimicrobial effects of a novel Triclosan/zinc citrate dentifrice against mixed culture oral biofilms. Int. Dent. J. 53(6Suppl 1), 371-378 (2003).

10. Wolff, M. S. \& Larson, C. The cariogenic dental biofilm: good, bad or just something to control. Braz. Oral. Res. 23(Suppl 1), 31-38 (2009).

11. Beighton, D. Can the ecology of the dental biofilm be beneficially altered? Adv. Dent. Res. 21, 69-73 (2009).

12. Lenander-Lumikari, M., Tenovuo, J. \& Mikola, H. Effects of a lactoperoxidase system-containing toothpaste on levels of hypothiocyanite and bacteria in saliva. Caries Res. 27, 285-291 (1993).

13. Tenovuo, J. Clinical applications of antimicrobial host proteins lactoperoxidase, lysozyme and lactoferrin in xerostomia: efficacy and safety. Oral. Dis. 8, 23-29 (2002).

14. Vertes, A., Hitchins, V. \& Phillips, K. S. Analytical challenges of microbial biofilms on medical devices. Anal. Chem. 84, 3858-3866 (2012). 
The antibacterial efficacy of a foam mouthwash and its ability to remove... SB Jones et al.

15. Plakunov, V. K., Martyanov, S. V., Teteneva, N. A. \& Zhurina, M. V. A universal method for quantitative characterization of growth and metabolic activity of microbial biofilms in static models. Microbiology 85, 509-513 (2016).

16. Zhou, H., Li, F., Weir, M. D. \& Xu, H. H. K. Dental plaque microcosm response to bonding agents containing quaternary ammonium methacrylates with different chain lengths and charge densities. J. Dent. 41, 1122-1131 (2013).

17. Jiang, H., Tai, B., Du, M. \& Peng, B. Effect of professional application of APF foam on caries reduction in permanent first molars in $6-7$ year old children: 24 month clinical trial. J. Dent. 33, 469-473 (2015).

18. Pessan, J. P., Toumba, K. J. \& Buzalaf, M. A. R. in Fluoride and the Oral Environment, Vol 22 (ed. Buzalaf, M. A. R.) 115-132 (Karger, Basel, 2011).

19. Rodriguez, E., Seguer, J., Rocabayera, X. \& Manresa, A. Cellular effects of monohydrochloride of L-arginine, Na-lauroyl ethylester (LAE) on exposure to Salmonella typhimurium and Staphylococcus aureus. J. Appl. Microbiol. 96, 903-912 (2004).

20. Kanazawa, A., Ikeda, T. \& Endo, T. A novel-approach to mode of action of cationic biocides-morphological effect on antibacterial activity. J. Appl. Bacteriol. 78, 55-60 (1995).

21. Ma, N., Chalmers, J. J., Auniņs, J. G., Zhou, W. \& Xie, L. Quantitative studies of cellbubble interactions and cell damage at different pluronic F-68 and cell concentrations. Biotechnol. Prog. 20, 1183-1191 (2004).

22. Chalmers, J. J. Gas bubbles and their influence on microorganisms. Appl. Mech. Rev. 51, 113-120 (1998).
23. Hannig, C., Spies, B., Spitzmüller, B. \& Hannig, M. Efficacy of enzymatic mouth rinses for immobilisation of protective enzymes in the in situ pellicle. Arch. Oral. Biol. 55, 1-6 (2010).

24. Delgado, A. J., Olafsson, V. G. \& Donovan, T. E. pH and erosive potential of commonly used oral moisturizers. J. Prosthodont. 25, 39-43 (2016).

(i) Open Access This article is licensed under a Creative Commons Attribution 4.0 International License, which permits use, sharing, adaptation, distribution and reproduction in any medium or format, as long as you give appropriate credit to the original author(s) and the source, provide a link to the Creative Commons license, and indicate if changes were made. The images or other third party material in this article are included in the article's Creative Commons license, unless indicated otherwise in a credit line to the material. If material is not included in the article's Creative Commons license and your intended use is not permitted by statutory regulation or exceeds the permitted use, you will need to obtain permission directly from the copyright holder. To view a copy of this license, visit http://creativecommons. org/licenses/by/4.0/.

(c) The Author(s) 2018 